\title{
Patent Ductus Venosus and Congenital Heart Disease: A Case Report and Review
}

\author{
Rachel Stork Poeppelman ${ }^{\mathrm{a}, \mathrm{d}}$, Joseph D. Tobias ${ }^{\mathrm{b}, \mathrm{c}}$
}

\begin{abstract}
In utero, the ductus venosus connects the left portal vein to the inferior vena cava, allowing a portion of the venous blood to bypass the liver and return to the heart. After birth, the ductus venosus closes due to changes in intracardiac pressures and a decrease in endogenous prostaglandins. Failure of the ductus venosus to close may result in galactosemia, hypoxemia, encephalopathy with hyperammonia, and hepatic dysfunction. We report an infant with complex congenital heart disease (CHD) who developed coagulopathy and hyperammonia during the preoperative period secondary to patent ductus venosus (PDV). Previous reports of PDV in CHD are presented, its etiology and clinical consequences reviewed, and options for therapeutic treatment discussed.
\end{abstract}

Keywords: Patent ductus venosus; Congenital heart disease; Portosystemic shunt

\section{Introduction}

Patent ductus venosus (PDV) is an uncommon vascular malformation, classified as a type of intrahepatic shunt [1]. In utero, the ductus venosus connects the left portal vein to the inferior vena cava, allowing a portion of the venous blood to bypass the liver and return to the heart. After birth, the ductus venosus generally closes between days of life 2 to 18 in term infants [2-5]. The natural decrease in portal venous pressure after birth contributes to the closure process [6-8]. Prostaglandins play a role in maintaining the patency of the ductus venosus while a product of the cytochrome P-450 system is postulated to con-

Manuscript submitted September 12, 2018, accepted September 26, 2018

aDivision of Pediatric Critical Care Medicine, Department of Pediatrics, The Ohio State University College of Medicine, Columbus, OH, USA

bDepartment of Anesthesiology and Pain Medicine, Nationwide Children's Hospital, Columbus, OH, USA

'Department of Anesthesiology and Pain Medicine, The Ohio State University College of Medicine, Columbus, OH, USA

${ }^{\mathrm{d} C}$ Corresponding Author: Rachel Poeppelman, Division of Pediatric Critical Care Medicine, Department of Pediatrics, Nationwide Children's Hospital, 700 Children's Drive, Columbus, OH 43205, USA.

Email: Rachel.Poeppelman@Nationwidechildrens.org

doi: https://doi.org/10.14740/cr777w tribute to its constriction $[2,9-12]$. If the ductus venosus fails to close, clinical signs may include galactosemia, hypoxemia, encephalopathy with hyperammonia, and hepatic dysfunction with disturbances of coagulation function and hyperbilirubinemia [1]. We report an infant with complex congenital heart disease (CHD) who developed coagulopathy and hyperammonemia during the preoperative period secondary to PDV. Once the diagnosis was made, hybrid stage 1 palliation was performed to allow for discontinuation of the prostaglandin infusion and facilitate PDV closure. Previous reports of PDV in CHD are presented, its etiology and clinical consequences reviewed, and options for therapeutic treatment discussed.

\section{Case Report}

Institutional Review Board approval is not required for single case reports at Nationwide Children's Hospital (Columbus, Ohio). A full term, $3 \mathrm{~kg}$ male infant presented in cardiogenic shock with poor perfusion on the second day of life. Workup including echocardiography revealed a type B interrupted aortic arch with a large malpositioned ventricular septal defect (VSD) and a patent ductus arteriosus (PDA). On admission to the cardiac intensive care unit, the capillary blood gas showed a $\mathrm{pH}$ of $6.92, \mathrm{PaCO} 222 \mathrm{~mm} \mathrm{Hg}$, and a base deficit of 27 $\mathrm{mmol} / \mathrm{L}$. Initial laboratory values were notable for coagulopathy and hyperammonemia $(107 \mu \mathrm{mol} / \mathrm{L})$ with signs of shock liver (elevated AST and ALT to $1,124 \mathrm{U} / \mathrm{L}$ and $526 \mathrm{U} / \mathrm{L}$ respectively). The patient's trachea was intubated, a prostaglandin E (PGE) infusion was started, and resuscitation provided by the administration of isotonic fluid and sodium bicarbonate. After initial resuscitation and initiation of the PGE infusion, his clinical status and laboratory parameters steadily improved. A left lower extremity catheter-associated deep vein thrombosis was noted and therapeutic unfractionated heparin therapy was started. On day of life (DOL) 12, worsening coagulation function was noted with a prothrombin time of $18.9 \mathrm{~s}$, INR of 1.63 , and hyperammonemia $(133 \mu \mathrm{mol} / \mathrm{L})$. The AST/ALT and total bilirubin did not significantly increase at this time; however, intermittent hypoglycemia was noted despite a glucose infusion rate of $10 \mathrm{mg} / \mathrm{kg} / \mathrm{min}$. On DOL 13, the serum lactate increased and the patient's trachea was reintubated. Planned surgical intervention was delayed due to the hepatic dysfunction and clinical decompensation. Since an ultrasound showed that the femoral vein thrombosis was non-occlusive and had not increased in size, the heparin infusion was discontinued on DOL 14. The workup for hepatic dysfunction and coagu- 


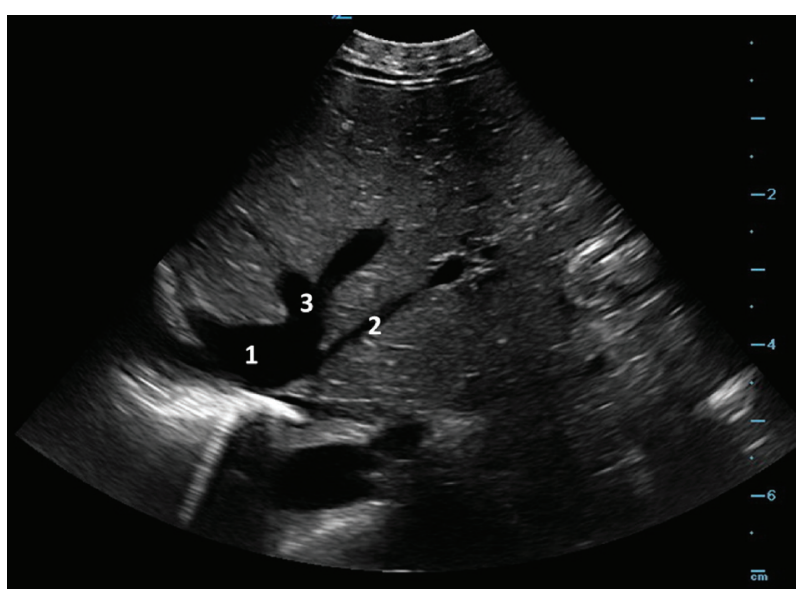

Figure 1. Hepatic and abdominal ultrasound showing hepatic vein confluence with the inferior vena cava (1); patent ductus venosus (2); and hepatic vein (3).

lopathy included a normal ferritin to rule out neonatal hemochromatosis, negative herpes simplex virus polymerase chain reaction, and a normal single nucleotide polymorphism microarray. A normal factor VIII level ruled out a consumptive process while low factor V and factor VII levels were consistent with decreased hepatic production. An abdominal ultrasound demonstrated a large patent ductus venosus with steal of blood flow from the portal vein and portosystemic shunting (Fig. 1). This was determined to be the cause of his hepatic dysfunction, coagulation disturbance, and hyperammonemia. As there was concern that the PGE infusion and high central venous pressures related to the CHD were contributing to the patency of the ductus venosus, the decision was made to proceed with surgical intervention. The coagulation disturbance was treated with fresh frozen plasma and he was taken to the operating room for a hybrid stage 1 palliation procedure on DOL 17. The PGE infusion was discontinued at the time of the procedure. Postoperatively, the coagulation disturbance, hypoglycemia, and hyperammonemia resolved. Follow-up ultrasound on DOL 21 showed a decrease in the size of the ductus venosus and by 6 weeks of age, the PDV had closed.

\section{Discussion}

Various factors may be responsible for hepatic dysfunction and its resultant laboratory alterations (hyperammonemia, hyperbilirubinemia, and coagulation disturbances) in the critically ill term neonate with CHD. Our patient presented in shock with end-organ dysfunction which could explain the associated hepatic dysfunction early in his clinical course. However, the hyperammonemia, hypoglycemia, and worsening coagulation function on DOL 12 were not accompanied by other signs of shock or hemodynamic decompensation. They occurred at a time when his clinical status was improving. An extensive workup was negative except for an abdominal ultrasound which showed a PDV and portosystemic shunting of the venous blood. Shunting of blood across a PDV has been shown to impair ammonia detoxification, blood coagulation and regu- lation of serum bile acids, with the clinical impact dictated by the percentage of blood that is shunted [13, 14]. Although PDV is a rare cause of hepatic dysfunction in the neonate, it may occur in patients receiving PGE infusions or those with elevated right-sided filling pressures due to CHD. Clinical signs and symptoms include hepatic dysfunction with coagulation disturbances, hyperbilirubinemia, and hyperammonemia as noted in our patient.

Of the 106 reported cases of PDV that we found in our literature review, 26 were in patients who also had CHD [1, $6,7,15-28]$. Of the PDV cases reported in CHD patients, all patients presented in childhood, with either clinical symptoms or incidental findings noted on imaging. Nineteen of the 26 patients presented at less than 2 years of age. The most common presenting symptoms in this population were hyperammonemia with encephalopathy, hyperbilirubinemia, and galactosemia. Only two previous cases of PDV in CHD patients presented with coagulation disturbances (prolonged PT and PTT) $[1,20]$. Other presenting symptoms included liver tumors, pulmonary hypertension (not secondary to CHD), elevated AST/ALT, developmental delay and membranoproliferative glomerulonephritis. The most common types of CHD associated with PDV included ventricular septal defect, atrial septal defect, and patent ductus arteriosus, although several previous case reports do not specify the type of CHD. Management strategies varied between conservative management (dietary restrictions and lactulose to treat hyperammonemia), endovascular closure, surgical ligation, and liver transplantation. Seven of the patients were reported to have died, most often due to cardiac complications from their CHD.

There is a recognized association between extrahepatic shunts and CHD [29]. Many authors have also speculated regarding the association of PDV, an intrahepatic shunt, and CHD $[15,17,18]$. Fugelseth et al demonstrated that high rightsided pressures due to CHD or pulmonary hypertension may lead to shunting through a PDV, potentially maintaining ductus venosus patency or contributing to its clinical significance [3]. Additionally, prostaglandins have been shown to play a role in maintaining the patency of the ductus venosus [2, 9-12]. Thus, in our patient, both the prostaglandin infusion and increased right-sided pressures may have contributed to ductus venosus patency, illustrating two factors which could place CHD patients at a higher risk for clinically significant PDV.

A PDV rarely closes spontaneously and is the most likely of the intrahepatic shunts to require surgical closure or a catheter-based intervention [30]. Alternatively, patients with CHD may require a palliative procedure or full correction of their $\mathrm{CHD}$ to lower right sided pressures and permit discontinuation of the PGE infusion to encourage closure of the PDV. Given the reported low likelihood of spontaneous closure of PDV, the spontaneous closure of our patient's PDV off PGE supports our assumption that the PGE infusion was contributing to patency of the ductus venosus. We would speculate that patients with a PDV on a PGE infusion, especially those with elevated right sided pressures, may experience a higher rate of spontaneous resolution than other PDV patients once the PGE infusion is discontinued. However, if a palliative procedure or full correction of the CHD is not feasible, surgical or catheter-based intervention may be necessary for closure of a PDV in select 
CHD patients, including those with persistent clinical manifestations. As noted by other authors, the adequacy of the portal venous system must be assessed before closure is attempted, both through imaging of the portal venous system and measurement of the rise in portal venous pressure with temporary occlusion of the PDV $[1,14]$. If a PDV is closed without an adequate portal venous system the patient can develop acute portal venous congestion and bowel ischemia [31].

Given the likely effect of PGE infusion and elevated rightsided pressures on the ductus venosus, a PDV is an important consideration in the differential diagnosis of hepatic dysfunction in neonates with CHD presenting with hyperbilirubinemia, coagulation disturbances or hyperammonemia. We would suggest a management strategy that minimizes factors contributing to PDV patency, such as a PGE infusion or high rightsided pressures, to encourage spontaneous closure of the PDV. This may require modification of the surgical plan for repair or palliation of CHD. When this is not possible, interventional or surgical closure of the PDV may be necessary if the portal venous system is found to be adequate.

\section{Conflict of Interest}

The authors have no conflict of interest to report.

\section{References}

1. Franchi-Abella S, Branchereau S, Lambert V, Fabre M, Steimberg C, Losay J, Riou JY, et al. Complications of congenital portosystemic shunts in children: therapeutic options and outcomes. J Pediatr Gastroenterol Nutr. 2010;51(3):322-330.

2. Fugelseth D, Lindemann R, Liestol K, Kiserud T, Langslet A. Postnatal closure of ductus venosus in preterm infants $<$ or $=32$ weeks. An ultrasonographic study. Early Hum Dev. 1998;53(2):163-169.

3. Fugelseth D, Kiserud T, Liestol K, Langslet A, Lindemann R. Ductus venosus blood velocity in persistent pulmonary hypertension of the newborn. Arch Dis Child Fetal Neonatal Ed. 1999;81(1):F35-39.

4. Kondo M, Itoh S, Kunikata T, Kusaka T, Ozaki T, Isobe $\mathrm{K}$, Onishi S. Time of closure of ductus venosus in term and preterm neonates. Arch Dis Child Fetal Neonatal Ed. 2001;85(1):F57-59.

5. Loberant N, Barak M, Gaitini D, Herskovits M, BenElisha M, Roguin N. Closure of the ductus venosus in neonates: findings on real-time gray-scale, colorflow Doppler, and duplex Doppler sonography. AJR Am J Roentgenol. 1992;159(5):1083-1085.

6. Maisawa S, Takasago Y, Oyake Y, Maeta H, Fujiwara T. Patent ductus venosus with hypoplastic right hepatoportal system in a young child born with asymmetric intra-uterine growth retardation. Eur J Pediatr. 1992;151(8):569571.

7. Mori K, Dohi T, Yamamoto H, Kamada M. An enormous shunt between the portal and hepatic veins associated with multiple coronary artery fistulas. Pediatr Radiol.
1990;21(1):66-68.

8. Meyer WW, Lind J. The ductus venosus and the mechanism of its closure. Arch Dis Child. 1966;41(220):597605.

9. Adeagbo AS, Breen CA, Cutz E, Lees JG, Olley PM, Coceani F. Lamb ductus venosus: evidence of a cytochrome P-450 mechanism in its contractile tension. J Pharmacol Exp Ther. 1990;252(2):875-879.

10. Coceani F. The control of the ductus venosus: an update. Eur J Pediatr. 1993;152(12):976-977.

11. Morin FC, 3rd. Prostaglandin E1 opens the ductus venosus in the newborn lamb. Pediatr Res. 1987;21(3):225228.

12. Takeuchi D, Momma K. Effect of decreased umbilical blood flow and hemorrhage, and decreased prostaglandins on the ductus venosus diameter in the rat. Biol Neonate. 2006;89(1):42-49.

13. Murayama K, Nagasaka H, Tate K, Ohsone Y, Kanazawa M, Kobayashi K, Kohno Y, et al. Significant correlations between the flow volume of patent ductus venosus and early neonatal liver function: possible involvement of patent ductus venosus in postnatal liver function. Arch Dis Child Fetal Neonatal Ed. 2006;91(3):F175-179.

14. Bernard O, Franchi-Abella S, Branchereau S, Pariente D, Gauthier F, Jacquemin E. Congenital portosystemic shunts in children: recognition, evaluation, and management. Semin Liver Dis. 2012;32(4):273-287.

15. Gitzelmann R, Arbenz UV, Willi UV. Hypergalactosaemia and portosystemic encephalopathy due to persistence of ductus venosus Arantii. Eur J Pediatr. 1992;151(8):564568.

16. Araki T, Kamada M, Okamoto Y, Arai S, Oba O. Coil embolization of a patent ductus venosus in a 52-dayold girl with congenital heart disease. Ann Thorac Surg. 2003;75(1):273-275.

17. Stringer MD. The clinical anatomy of congenital portosystemic venous shunts. Clin Anat. 2008;21(2):147-157.

18. Paley MR, Farrant P, Kane P, Heaton ND, Howard ER, Karani JB. Developmental intrahepatic shunts of childhood: radiological features and management. Eur Radiol. 1997;7(9):1377-1382.

19. Ohno T, Muneuchi J, Ihara K, Yuge T, Kanaya Y, Yamaki S, Hara T. Pulmonary hypertension in patients with congenital portosystemic venous shunt: a previously unrecognized association. Pediatrics. 2008;121(4):e892-899.

20. Bellah RD, Hayek J, Teele RL. Anomalous portal venous connection to the suprahepatic vena cava: sonographic demonstration. Pediatr Radiol. 1989;20(1-2):115-117.

21. Gillespie MJ, Golden A, Sivarajan VB, Rome JJ. Transcatheter closure of patent ductus venosus with the Amplatzer vascular plug in twin brothers. Pediatr Cardiol. 2006;27(1):142-145.

22. Takehara Y, Mori K, Edagawa T, Sugimoto M, Takehara H, Ito M, Kuroda Y. Presumed hypoplastic intrahepatic portal system due to patent ductus venosus: Importance of direct occlusion test of ductus venosus under open laparotomy. Pediatr Int. 2004;46(4):484-486.

23. Eroglu Y, Donaldson J, Sorensen LG, Vogelzang RL, Melin-Aldana H, Andersen J, Whitington PF. Improved 
neurocognitive function after radiologic closure of congenital portosystemic shunts. J Pediatr Gastroenterol Nutr. 2004;39(4):410-417.

24. Mitchell IM, Pollock JC, Gibson AA. Patent ductus venosus. Pediatr Cardiol. 1991;12(3):181-183.

25. Sanada Y, Urahashi T, Ihara Y, Wakiya T, Okada N, Yamada N, Egami S, et al. The role of operative intervention in management of congenital extrahepatic portosystemic shunt. Surgery. 2012;151(3):404-411.

26. Ihara K, Hijii T, Kuromaru R, Ariyoshi M, Kira R, Fukushige J, Hara T. High-intensity basal ganglia lesions on T1-weighted images in two toddlers with elevated blood manganese with portosystemic shunts. Neuroradiology. 1999;41(3):195-198.

27. Schierz IA, La Placa S, Giuffre M, Montalbano G, Len- zo M, Corsello G. Transient hepatic nodular lesions associated with patent ductus venosus in preterm infants. Am J Perinatol. 2011;28(3):177-180.

28. Farrant P, Meire HB, Karani J. Ultrasound diagnosis of portocaval anastomosis in infants - a report of eight cases. Br J Radiol. 1996;69(821):389-393.

29. Massin M, Verloes A, Jamblin P. Cardiac anomalies associated with congenital absence of the portal vein. Cardiol Young. 1999;9(5):522-525.

30. Paganelli M, Lipsich JE, Sciveres M, Alvarez F. Predisposing factors for spontaneous closure of congenital portosystemic shunts. J Pediatr. 2015;167(4):931-935 e912.

31. Barsky MF, Rankin RN, Wall WJ, Ghent CN, Garcia B. Patent ductus venosus: problems in assessment and management. Can J Surg. 1989;32(4):271-275. 